\title{
A RELAÇÃO EXISTENTE ENTRE O PROCESSO DE ACREDITAÇÃO E A ASSISTÊNCIA FARMACÊUTICA HOSPITALAR
}

\section{ARTIGO DE REVISÃO}

DIAS, Rayssa Rodrigues ${ }^{1}$

DIAS, Rayssa Rodrigues. A relação existente entre o processo de acreditação e a assistência farmacêutica hospitalar. Revista Científica Multidisciplinar Núcleo do Conhecimento. Ano 05, Ed. 05, Vol. 01, pp. 104-120. Maio de 2020. ISSN: 2448-0959, Link de acesso: https://www.nucleodoconhecimento.com.br/saude/processo-de$\underline{\text { acreditacao }}$

\section{RESUMO}

Os hospitais têm adotado programas de gestão da qualidade com o objetivo de melhorar, de forma contínua, os processos, produtos e serviços, utilizando-se da acreditação como método de avaliação externa da qualidade em serviços de saúde. O processo de acreditação em unidades hospitalares é um tema relativamente recente e por isso quando se realiza a busca sobre o assunto na internet poucos artigos são encontrados. Estima-se que apenas $5 \%$ dos hospitais brasileiros sejam acreditados, sendo a maioria de natureza privada. Este artigo trata da relação existente entre a assistência farmacêutica hospitalar e o processo de acreditação. A metodologia utilizada foi a pesquisa bibliográfica, onde foram selecionados artigos científicos atuais, legislações, dentre outros documentos com foco no cenário brasileiro, a fim de obter-se uma revisão do assunto tratado. Concluiu-se que o emprego das boas práticas da qualidade em serviços de saúde nos hospitais, principalmente quando tratamos da assistência farmacêutica prestada pelo setor de farmácia, associada à acreditação, proporciona inúmeros benefícios dos quais podemos citar a maior produtividade, aumento na satisfação do paciente e valorização da instituição, porém

${ }^{1}$ Graduação em Farmácia; Especialização em Análises Clínicas e Microbiologia; Especialização em Farmácia Hospitalar e Mestrado em Ciências. 
a grande problemática envolvida é a adesão dos hospitais brasileiros à esse novo conceito de avaliação devido ao alto custo gerado para a unidade de saúde e pelo fato de se tratar de um processo voluntário.

Palavras-Chave: Farmacêutico, farmácia hospitalar, qualidade, acreditação hospitalar, assistência farmacêutica.

\section{INTRODUÇÃO}

A Assistência Hospitalar é uma das áreas mais estudadas ou mesmo discutidas na atualidade devido ao seu grande impacto na vida da população em geral. No Brasil sua implementação é focada no uso de indicadores de qualidade, sendo cada vez mais utilizada e valorizada na gestão hospitalar. Seus critérios de avaliação, construção e acompanhamento ainda não estão bem definidos já que não existe uma única metodologia.

Quando se trata de qualidade em serviços de saúde, o controle de gestão hospitalar é um fator de grande relevância para a eficiência de uma unidade. É necessário que os gestores realizem uma avaliação do desempenho organizacional, a fim de mensurar a eficiência da gestão. Uma das alternativas que possibilitam a mensuração do controle da gestão em organizações hospitalares é o processo de Acreditação Hospitalar, um sistema de avaliação e certificação da qualidade de serviços de saúde por meio de padrões previamente aceitos que se utiliza de indicadores de qualidade.

Uma das barreiras que leva à não adesão dos hospitais brasileiros ao processo de acreditação é o alto custo. Outro fato é a questão de não haver garantia de que a acreditação afete diretamente a qualidade, apenas indica que o hospital deseja melhorar, então os hospitais não possuem interesse em se acreditar já que não existe nenhuma obrigatoriedade por se tratar de um processo voluntário, ou seja, de livre escolha da organização de saúde, ao contrário do que acontece em outros países onde a implementação da acreditação é obrigatória prevista em legislação. 


\section{OBJETIVOS}

O trabalho tem como objetivo geral elucidar o vínculo existente entre a assistência farmacêutica hospitalar e o processo de acreditação, abrangendo de forma generalista a atual situação no Brasil.

Como objetivos específicos apresentar alguns conceitos, relacionar as acreditações nacionais e internacionais focalizando no cenário brasileiro, determinar formas de avaliação da qualidade, breve histórico do manual de acreditação, citar os indicadores de desempenho e esclarecer a importância destes que são comumente utilizados na avaliação da assistência farmacêutica.

\section{JUSTIFICATIVA}

O artigo justifica-se na escassez de conteúdo sobre o assunto na literatura, já que a maioria dos hospitais brasileiros ainda não realizou o processo de acreditação hospitalar para a avaliação do desempenho da assistência farmacêutica. Desse modo, destaca-se a importância de se reunir informações acerca do assunto. É estimado que apenas $5 \%$ dos estabelecimentos hospitalares brasileiros são acreditados, sendo que 40\% deles estão localizados em São Paulo. Em contrapartida, nos Estados Unidos da América (EUA) mais de $90 \%$ dos hospitais têm acreditações. O processo de acreditação no território nacional é uma iniciativa que predomina em instituições privadas e nota-se que, os hospitais públicos acreditados oferecem uma assistência qualificada a seus usuários que se assemelha a dos hospitais privados. De acordo com uma entrevista dada em 2015 por Ana Maria Malik, coordenadora do núcleo de saúde da Fundação Getúlio Vargas, à folha UOL, não há vantagem em ser acreditado no Brasil bem como não existe penalidade por não o ser. Pode ser por esse motivo, somado ao alto custo do processo, que os hospitais não executam a ação de implementar a acreditação. Ela pode ser vantajosa em termos de padronização dos procedimentos envolvidos, na contenção dos gastos e até mesmo na mercadologia, caso a acreditação venha a ser considerada como indicador da qualidade pelo público-alvo. 


\section{METODOLOGIA}

A metodologia utilizada foi a pesquisa bibliográfica, que procura elucidar ou discutir um determinado assunto com base em referências teóricas confiáveis, não configurando apenas a transcrição dos fatos encontrados nos documentos pesquisados. Utilizou-se de artigos científicos atuais em português e outros tipos de materiais com o intuito de obter abrangência sobre o assunto. $O$ que se restringiu foi apenas a área geográfica (Brasil) para que através da investigação da problemática pudéssemos entender em que grau o país se encontra em relação a esse assunto considerado tanto importante como novo.

\section{DESENVOLVIMENTO}

\subsection{A ACREDITAÇÃO HOSPITALAR}

A acreditação, que é um sistema de avaliação e certificação da qualidade de serviços de saúde por meio de padrões previamente aceitos e realizada por uma organização especializada e independente, reflete as origens da avaliação sistemática de determinados países. É um processo voluntário, reservado e que ocorre dentro de uma determinada periodicidade para verificar se a instituição segue rígidos padrões de segurança estabelecidos por acreditadoras competentes, nacionais ou internacionais. Os padrões podem ser mínimos ou mais elaborados e exigentes, o que definirá os diferentes níveis de satisfação e qualificação. Objetiva estimular o desenvolvimento de uma cultura de melhoria contínua da qualidade na assistência médico-hospitalar e na proteção à saúde da população (SHAW, 2000) (RODRIGUES e TUMA, 2011) (EBSERH, 2019).

Diversos autores relatam como benefícios diretos do processo de acreditação a qualidade da assistência; a segurança para os pacientes e profissionais; construção de uma equipe multidisciplinar; útil instrumento de gerenciamento; o caminho para a melhoria contínua; credibilidade junto à população; gerenciamento por indicadores. É uma ação coordenada por uma organização ou agência não governamental encarregada do desenvolvimento e implantação de sua própria metodologia. A 
conquista da acreditação simboliza que a instituição segue normas técnicas rígidas que asseguram segurança e qualidade tanto para os profissionais quanto para os pacientes.

\subsection{A ACREDITAÇÃO HOSPITALAR NO BRASIL}

A acreditação hospitalar no Brasil é recente. Ainda que as primeiras iniciativas de avaliação da qualidade dos serviços de saúde tenham sido feitas na década de setenta, foi após a criação do Programa Brasileiro de Acreditação Hospitalar (PBAH) na década de noventa, que a acreditação ganhou maior projeção. O PBAH é operacionalizado pela Organização Nacional de Acreditação (ONA), responsável pela difusão da acreditação entre as organizações de saúde, incluindo os hospitais (ORGANIZAÇÃO NACIONAL DE ACREDITAÇÃO, 2013). Estima-se que menos que $5 \%$ dos hospitais brasileiros possuam o certificado de acreditação (FOLHA DE SÃO PAULO, 2015).

No Brasil, a acreditação hospitalar vem sendo discutida desde o ano de 1990, sob influência da OPAS/OMS e o foco principal era o desenvolvimento da infraestrutura de saúde, sendo as primeiras iniciativas voltadas para a realidade hospitalar (FORTES e FARIA, 2012). Diversas iniciativas destacam-se dentro de um movimento precursor de avaliação dos serviços de saúde com algum instrumento constituído por padrões, como o Programa de Avaliação e Certificação de Qualidade em Serviços de Saúde, no Rio de Janeiro; o Instituto de Administração Hospitalar localizado no Rio Grande do Sul e o Instituto Brasileiro de Acreditação Hospitalar em São Paulo, dentre outros. O Ministério da Saúde percebeu a necessidade de se criar uma ferramenta nacional para a avaliação e certificação de serviços de saúde, com isso foi instituída uma comissão nacional de especialistas para o desenvolvimento do modelo brasileiro de acreditação, momento este onde elaborou-se a primeira versão do Manual Brasileiro de Acreditação Hospitalar (RODRIGUES e TUMA, 2011) (ALONSO et al, 2014). 


\subsection{HISTÓRICO DO MANUAL BRASILEIRO DE ACREDITAÇÃO SEGUNDO A ONA}

Em 1998, quando foi publicada a primeira versão do Manual Brasileiro de Acreditação, a classificação dava-se em quatro (4) níveis. Já na segunda edição foram instituídos três (3) níveis de acreditação visto que o nível quatro foi excluído, onde o nível 1 era denominado "acreditado" (ONA 1), o nível 2 "acreditado pleno" (ONA 2) e o nível 3 "acreditado com excelência" (ONA 3). Com o passar dos anos, os padrões preconizados para o nível 1 foram sendo incorporados às unidades hospitais. Hoje, para alcançar o nível 1 e os demais níveis as exigências são completamente diferentes do que era previsto há duas décadas.

Em 2001, a ONA lança sua primeira publicação, o Manual das Organizações Prestadoras de Serviços Hospitalares/ Organização Nacional de Acreditação - ONA - Coleção Manual Brasileiro de Acreditação - Volume 1. A partir desse ano, são firmados convênios com a ANVISA que demandou-se que fossem elaborados padrões e manuais que pudessem ser utilizados em outros tipos de estabelecimentos.

A partir disso, em 2003, tem-se o lançamento do Manual de Acreditação das Organizações Prestadoras de Serviço de Hemoterapia, que foi o primeiro concluído e publicado, resultante do convênio entre ANVISA e ONA.

Posteriormente, é lançado o Manual de Acreditação das Organizações Prestadoras de Serviços de Laboratórios Clínicos, que foi a primeira publicação destinada aos laboratórios, fruto de uma parceria firmada entre ANIVSA, ONA, Sociedade Brasileira de Análises Clínicas (SBAC) e Sociedade Brasileira de Patologia Clínica (SBPC).

Já em 2003 é lançado o Manual de Acreditação das Organizações Prestadoras de Serviços de Nefrologia e Terapia Renal Substitutiva, resultado de uma parceria com a Sociedade Brasileira de Nefrologia (SBN). 
Em 2006, a ONA lançou e agrupou seus manuais em áreas como: serviços de hemoterapia, laboratório clínico, nefrologia e terapia renal, hospitalares, radioterapia e serviços ambulatoriais.

Em 2010, os manuais são consolidados em uma única publicação, e ainda houve a aprovação do Manual de Acreditação de Programas de Promoção da Saúde e Prevenção de Riscos e Doenças pela Agência Nacional de Saúde Suplementar (ANS), o que foi considerado mais um marco na área.

Em 2011, os serviços adjacentes ao segmento de saúde passam a ser certificados com o Selo de Qualificação ONA com o lançamento do Manual para Avaliação e Certificação dos Serviços para a Saúde, que abrangia áreas como lavanderia, esterilização e reprocessamento de materiais, serviço de manipulação de drogas antineoplásicas e de dietas parenterais e serviços de dietoterapia.

Em 2012, de uma parceria entre a ONA e as Instituições Acreditadoras Credenciadas (IACs), a Faculdade de Odontologia da Universidade de São Paulo (USP) e a Associação Brasileira de Cirurgiões-Dentistas, os serviços odontológicos ganharam um manual.

Em 2013, o manual consolidado recebe a chancela da International Society for Quality in Health Care (ISQua) e, em 2014, ocorre o lançamento da edição do manual certificada pelo órgão internacional.

A versão mais atual do referido manual é do ano de 2018, e foi novamente submetida e referendada pela ISQua, que certificada acreditadoras que seguem padrões internacionalmente reconhecidos (ONA, 2020).

\subsection{ACREDITAÇÕES NACIONAIS E INTERNACIONAIS}

Em relação às acreditações nacionais têm-se a Organização Nacional de Acreditação (ONA), o Programa de Controle da Qualidade Hospitalar (CQH) e o Prêmio Nacional de Gestão em Saúde (PNGS). A difusão da acreditação hospitalar no Brasil é de responsabilidade da ONA. 
Dentre as acreditações internacionais e estrangeiras temos a Joint Commission International - JCI (internacional), Canadian Council on Health Services Accreditation - CCHSA (canadense) e National Integrated Accreditation for Healthcare Organizations - NIAHO (norueguesa) (RODRIGUES; TUMA, 2011) (MV, 2018). O Consórcio Brasileiro de Acreditação é o único no Brasil associado à $\mathrm{JCl}$ através de um acordo de cooperação que já existe há 20 anos (CBA, 2020).

Existem também as certificações, como a ISO 9000 e a norma OHSAS 18001. A ISO 9000 é considerada a rede mundial de institutos de padronização mais importante. $O$ conjunto composto pelas normas 9000, 9001, 9004 e 9011 relacionam-se às orientações básicas para a implementação dos sistemas de gestão da qualidade, controle de riscos, diretrizes para auditorias, entre outros. Já a OHSAS 18001, define os mínimos requisitos para práticas de excelência em saúde e segurança ocupacional. As acreditações e certificações se complementam, são primordiais para que uma unidade hospitalar alcance a excelência na prestação dos serviços de saúde, fortalecendo seu respeito perante os colaboradores, clientes e fornecedores (MV, 2018).

Quando a Agência Nacional de Vigilância Sanitária (ANVISA) propôs a estrutura do Conselho da ONA, visualizava uma organização tripartite com representação do Ministério da Saúde, das empresas "compradoras" de serviços hospitalares e representação dos órgãos daqueles que "vendem" estes serviços imaginando que com este tipo de estrutura, estas instituições iriam exigir de forma voluntária que os hospitais fossem acreditados para garantir a necessária qualidade da atenção aos seus clientes. Apesar dos avanços deste programa no Brasil nos últimos anos, ainda não é visto um maior número de hospitais acreditados como previa-se anteriormente (ANVISA, 2019).

Organizações de diversos portes, perfis e complexidades podem se tornar qualificadas ou acreditadas basta cumprir os padrões que são estabelecidos pela ONA. Para participar do processo de acreditação, a organização prestadora de serviços de saúde precisa cumprir alguns requisitos de elegibilidade que são exigidos como, por exemplo, possuir licença sanitária, alvará de funcionamento, licenças 
pertinentes à natureza da atividade, registro do responsável técnico conforme o perfil da organização e estar legalmente constituída há pelo menos 1 ano (ONA, 2019).

De acordo com o Mapa de Acreditações presente no site da ONA observa-se que do total das 877 certificações ativas a sua maioria pertence à hospitais (342) seguido pelos serviços laboratoriais (126); serviços ambulatoriais (117); serviços de diagnóstico por imagem, radioterapia e medicina nuclear (106); serviços oncológicos (46); serviços de hemoterapia (39); Hospital Dia (21); Serviços de Pronto Atendimento (13); Nefrologia-Crônica (9); Atenção Domiciliar-Assistência e Internação (8); Serviços de Nefrologia e Terapia Renal Substitutiva (8); Serviços de Manipulação (8); Serviços de Atenção Domiciliar (7); Nefrologia-Crônica e Aguda (6); Processamento de roupas para saúde (5); Serviço odontológico (5); Esterilização e Processamento de Materiais (3); Programas de Saúde e Prevenção de Riscos (3); Serviços de Medicina Hiperbárica (3); Atenção Domiciliar-Assistência (1) e Serviços de Dietoterapia (1) (ONA, 2020).

O valor da taxa cobrada no processo de avaliação para certificação no ano corrente (2020) em organizações prestadoras de serviços hospitalares e Hospital Dia varia de acordo com o número de leitos que o hospital possui, sendo $R \$ 46,00$ o valor por leito; $R \$ 2.300,00$ (taxa mínima) até 50 leitos; entre $R \$ 2.346,00$ a $R \$ 4.600,00$ de 51 a 100 leitos; de $R \$ 4.646,00$ à $R \$ 13.800,00$ de 101 a 300 leitos e a partir de $R \$ 13.846,00$ acima de 300 leitos (ONA, 2020). A principal causa do desinteresse da maioria dos hospitais no Brasil em relação ao processo de acreditação continua sendo o alto custo que é gerado para a unidade de saúde.

\subsection{NÍVEIS DE ACREDITAÇÃO DA ORGANIZAÇÃO NACIONAL DE ACREDITAÇÃO (ONA)}

De acordo com a acreditação ONA a instituição pode alcançar certificações em três diferentes níveis, conforme o cumprimento de padrões do Manual Brasileiro de Acreditação. Os níveis são crescentes e envolvem requisitos relacionados à estrutura (nível 1), processos (nível 2) e resultados (nível 3). O nível 1 é denominado acreditado, o nível 2 acreditado pleno e nível 3 acreditado com excelência. Os níveis 1 e 2 
fornecem certificado válido por 2 anos, já o nível 3 o certificado possui validade por 3 anos (ONA, 2019).

No nível 1 a organização de saúde deve cumprir ou superar, em $70 \%$ ou mais, os padrões de qualidade e segurança definidos por esta organização. Além dos aspectos estruturais e assistenciais, todas as áreas de atividades da instituição são avaliadas. No nível 2 a instituição precisará atender a 2 critérios: cumprir ou superar, em $80 \%$ ou mais, os padrões de qualidade e segurança ou cumprir ou superar, em $70 \%$ ou mais, os padrões da ONA de gestão integrada, com processos ocorrendo com fluidez e plena comunicação entre as atividades envolvidas. Já no nível 3, a organização precisará atender a 3 critérios que são: cumprir ou superar, em $90 \%$ ou mais, os padrões de qualidade e segurança; cumprir ou superar, em $80 \%$ ou mais, os padrões de gestão integrada; e cumprir ou superar, em $70 \%$ ou mais, os padrões ONA de Excelência em Gestão, de forma que demonstre uma cultura organizacional de melhoria contínua com maturidade institucional (ANVISA, 2004) (ONA, 2019).

\subsection{O PROFISSIONAL FARMACÊUTICO}

Diversos profissionais estão envolvidos no processo de Acreditação Hospitalar, um deles é o farmacêutico que é o "profissional da área de saúde, com formação centrada nos fármacos, nos medicamentos e na assistência farmacêutica e, de forma integrada, com formação em análises clínicas e toxicológicas, em cosméticos e em alimentos, em prol do cuidado à saúde do indivíduo, da família e da comunidade" conforme traz o documento das Diretrizes Curriculares Nacionais do Curso de Graduação em Farmácia, publicado pela Câmara de Educação Superior do Conselho Nacional de Educação (CNE), em 19 de outubro de 2017 (GUIA DA FARMÁCIA, 2018).

Segundo o Conselho Federal de Farmácia (CFF) esse profissional pode atuar em diversas áreas. A Resolução do CFF no 572, de 25 de abril de 2013, que trata das especialidades farmacêuticas, agrupa 10 linhas de atuação do farmacêutico incluindo as áreas de alimentos; análises clínico-laboratoriais; educação; farmácia; farmácia hospitalar e clínica; farmácia industrial; gestão; práticas integrativas e complementares; saúde pública e toxicologia (CFF, 2013). 
De acordo com o Instituto de Ciência, Tecnologia e Qualidade (ICTQ) a carreira farmacêutica especializada em farmácia hospitalar tem grande valorização no mercado de trabalho e permite ao profissional atuar tanto em hospitais filantrópicos como também em hospitais públicos e privados. Nas unidades hospitalares esse profissional é capacitado para realizar atividades de Atenção Farmacêutica a pacientes internados e ambulatoriais, visando o uso racional dos medicamentos; atuar na logística farmacêutica, tendo o medicamento como insumo mais importante; pode representar a farmácia nas comissões hospitalares, sendo o balizador de decisões em tudo que se refere ao medicamento; atuar como responsável legal pelo fluxo dos medicamentos dentro da unidade hospitalar; elaborar normas e controles que garantam a qualificação de fornecedores; é o responsável pela dispensação do medicamento por meio de sistemas que permitam fluxos racionais e que minimizem a ocorrência de erros; se responsabiliza também pelo plano de gerenciamento de resíduos de serviços de saúde (PGRSS) da farmácia e pela análise, comparação de custos e consequências das terapias medicamentosas aos pacientes; manipula fórmulas magistrais, oficinais e parenterais, de acordo com os princípios das boas práticas de manipulação em farmácia; é o responsável pelas atividades relacionadas ao controle de qualidade dos insumos recebidos; pelas ações de farmacovigilância dentro da farmácia hospitalar; tem participação efetiva nas comissões hospitalares e também cuida da elaboração de editais de compra e especificações técnicas para medicamentos e correlatos (ICTQ, 2015).

\subsection{FARMÁCIA HOSPITALAR}

A Portaria $n^{\circ} 4.283$ de 30 de dezembro de 2010 , que aprova as diretrizes e estratégias para organização, fortalecimento e aprimoramento das ações e serviços de farmácia no âmbito dos hospitais, conceitua Farmácia Hospitalar como a unidade clínicoassistencial, técnica e administrativa, onde são processadas atividades relacionadas à assistência farmacêutica, dirigida por profissional farmacêutico, compondo a estrutura organizacional do hospital e integrada com as unidades administrativas e de assistência ao paciente. A Farmácia Hospitalar é um setor de suma importância dentro 
do hospital devido aos elevados valores orçamentários que demanda para a unidade (MS, 2010).

Dentre as diversas atividades da Farmácia Hospitalar a que tem tido maior destaque é a Assistência Farmacêutica, pois é através dela que podem ser alcançados resultados significativos quando falamos de adesão do paciente à terapia medicamentosa, diminuição de erros na administração de medicamentos, uso racional de medicamentos, dentre outros benefícios.

\subsection{ASSISTÊNCIA FARMACÊUTICA E ACREDITAÇÃO HOSPITALAR}

A Resolução no 338, de 06 de maio de 2004, do Conselho Nacional de Saúde, define Assistência Farmacêutica como sendo:

[...] um conjunto de ações voltadas à promoção, proteção e recuperação da saúde, tanto individual como coletivo, tendo o medicamento como insumo essencial e visando o acesso e ao seu uso racional. Este conjunto envolve a pesquisa, o desenvolvimento e a produção de medicamentos e insumos, bem como a sua seleção, programação, aquisição, distribuição, dispensação, garantia da qualidade dos produtos e serviços, acompanhamento e avaliação de sua utilização, na perspectiva da obtenção de resultados concretos e da melhoria da qualidade de vida da população.

A mesma resolução também relata sobre as ações da Assistência Farmacêutica e a interação do farmacêutico com o usuário:

As ações de Assistência Farmacêutica envolvem aquelas referentes à Atenção Farmacêutica, considerada como um modelo de prática farmacêutica, desenvolvida no contexto da Assistência Farmacêutica e compreendendo atitudes, valores éticos, comportamentos, habilidades, compromissos e corresponsabilidades na prevenção de doenças, promoção e recuperação da saúde, de forma integrada à equipe de 
saúde. É a interação direta do farmacêutico com o usuário, visando uma farmacoterapia racional e a obtenção de resultados definidos e mensuráveis, voltados para a melhoria da qualidade de vida. Esta interação também deve envolver as concepções dos seus sujeitos, respeitadas as suas especificidades biopsicossociais, sob a ótica da integralidade das ações de saúde.

A direção técnica da Farmácia Hospitalar, na figura do farmacêutico, deve estar focada em prestar a Assistência Farmacêutica aos pacientes internados conforme a Política Nacional de Assistência Farmacêutica (PNAF), a Política Nacional de Medicamentos e demais normativas envolvidas, porém nem sempre isso é possível, ainda mais quando tratamos da estruturação da Assistência Farmacêutica no âmbito do Sistema Único de Saúde (SUS) (SBRAFH, 2017).

A garantia da qualidade envolvida no processo de Assistência Farmacêutica Hospitalar pode ser mensurada através do processo de acreditação hospitalar, onde é feita a implementação de indicadores de desempenho (SANTOS e LIMBERGER, 2018). Como exemplos desses indicadores de desempenho relativos à Assistência Farmacêutica Hospitalar temos: a padronização de medicamentos e produtos médicos; consumo médio mensal; estoque mínimo; ponto de ressuprimento ou ponto de pedido; devoluções de materiais e medicamentos; logística adequada de medicamentos; padronização de receituários e notas de sala; capacitação de recursos humanos; participação em comissões; cumprimento aos Procedimentos Operacionais Padrão (POPs); conciliação medicamentosa (SANTOS e LIMBERGER, 2018).

\subsection{INDICADORES DE DESEMPENHO DA ASSISTÊNCIA FARMACÊUTICA HOSPITALAR}

Os indicadores de desempenho são instrumentos utilizados para avaliação e melhoria de certos processos e exercem papel importante na demanda pela qualidade. Quando tratamos de farmácias hospitalares, os indicadores são cruciais para o planejamento e também o replanejamento de uma instituição e contribuem na diminuição de riscos e na contínua melhoria da assistência (SITEWARE, 2018). 
A seguir temos alguns dos indicadores de desempenho utilizados na área de farmácia hospitalar:

- Pacientes atendidos: considera o número de pacientes atendidos pela farmácia hospitalar. Os usuários são segregados como: caso novo, renovação e continuidade.

- Tempo de espera: avalia a rapidez do atendimento ao usuário da farmácia hospitalar, ou seja, o intervalo de tempo de espera para que o paciente seja efetivamente atendido.

- Solicitações indeferidas: objetiva quantificar e avaliar as principais causas que levaram à devolução ou indeferimento das solicitações de medicamentos.

- Satisfação do usuário: utiliza-se de pesquisas para se mensurar e avaliar o quão satisfeitos ou não os usuários estão com os serviços oferecidos pela farmácia hospitalar.

- Medicamento em falta: quantifica os medicamentos que faltaram no estoque da farmácia em determinado intervalo de tempo, sugerindo as possíveis causas do desabastecimento.

- Medicamentos não utilizados: relaciona-se os medicamentos que não foram utilizados e o custo que eles representam para a unidade hospitalar.

- Inventário geral de acuracidade: realizar um controle efetivo do estoque da farmácia de modo que haja concordância entre a quantidade de medicamento armazenada no estoque e a quantidade que consta no sistema computacional.

- Índice de notificações de reação adversa a medicamento: quantifica e acompanha o número de notificações que foram feitas sobre qualquer tipo de reação adversa ao medicamento utilizado.

- Entregas fora do prazo: determina a quantidade de medicamentos que foram entregues com atraso pelos fornecedores.

- Taxa de falta de medicamento ao paciente internado: mensurar quantos e quais medicamentos faltaram para os pacientes internados.

- Índice de treinamento de profissionais da farmácia: o uso desse indicador estima a quantidade de treinamentos realizados pelos profissionais da farmácia (SITEWARE, 2018). 


\subsection{A IMPORTÂNCIA DOS INDICADORES DE DESEMPENHO DA FARMÁCIA HOSPITALAR}

As unidades de saúde têm buscado avaliar e acompanhar os serviços prestados a fim de garantir a qualidade final para o usuário. Nesse sentido, os indicadores de desempenho indicados para uso na farmácia hospitalar estabelecem quais os padrões a serem seguidos, e a partir disso possibilitando que sejam feitas análises críticas e que sejam tomadas decisões mais condizentes com a situação em questão.

A implementação de indicadores de desempenho de farmácia hospitalar ajuda a prevenir erros de medicamentos, a promover o uso correto e racional destes e a minimizar custos terapêuticos e período de tratamento. Os indicadores são relevantes também para o controle dos processos internos e a utilização destes necessita de que estejam atrelados tanto à estratégia como aos processos críticos da sua instituição.

Desta forma, o uso sistemático permitirá que a farmácia tenha conhecimento sobre os seus pontos positivos e sobre suas dificuldades, facilitando a mensuração de estratégias de melhoria dos hábitos de trabalho e de melhoramento dos recursos financeiros e humanos.

Os indicadores de desempenho devem ser assistidos com determinada frequência, pois eles servirão como fundamento para identificar as reais necessidades e reavaliar possíveis estratégias.

Os softwares de gestão de performance corporativa são considerados a melhor forma de se acompanhar os indicadores pois permitem que as informações fluam de forma clara, integrando pessoas, estratégia e execução. Outra vantagem desse recurso é pelo fato de se tratar de um controle automatizado que possibilita o preparo para a conquista de certificações e acreditações hospitalares (SITEWARE, 2020). 


\section{DISCUSSÃO}

Ao entender melhor a importância dos indicadores de qualidade e seu papel na gestão da Assistência Farmacêutica fica evidente que esta ferramenta é de grande relevância, portanto, a avaliação de desempenho torna-se uma preocupação constante. Este desempenho envolve muitos aspectos que influenciam principalmente

na questão financeira e na qualidade do atendimento, ainda mais quando falamos da rede pública de saúde.

Se por um lado a acreditação é capaz de realizar um grande progresso, por outro encontra a dificuldade em sua inserção devido ao alto custo. O Brasil comparado a outros países está atrasado nessa questão e precisa reconhecer a importância do processo para fins de aplicação da acreditação e entender o quanto o processo pode levar a melhorias. Talvez fosse necessária a obrigatoriedade através de legislação específica para que os hospitais brasileiros pudessem chegar mais perto de países como os EUA, onde mais de $90 \%$ dos hospitais possuem a certificação.

Observa-se, portanto, que a implantação do sistema de qualidade não é tarefa simples, ao contrário, apresenta-se como um grande desafio a ser enfrentado pelos profissionais de saúde e gestores que buscam adequar sua rotina de trabalho a excelência no atendimento aos pacientes.

De acordo com um artigo publicado por Sack et al. (2011), descreveu-se uma pesquisa realizada junto a 37.777 pacientes que receberam alta de internação de 73 diferentes hospitais, entre unidades acreditadas e não acreditadas, na Alemanha, não evidenciou-se estatisticamente que o nível de satisfação se relaciona com a posse do certificado de acreditação. Tal fato considera a necessidade de incluir as verdadeiras emergências do paciente e de outros envolvidos no atendimento realizado pelos médicos.

Um número de unidades hospitalares considerado pouco expressivo, mas com tendência crescente, está avançando em se obter certificações e/ou acreditações em 
norma externa. Isso demonstra que a busca incessante pela qualidade tem crescido, ao menos na esfera dos hospitais privados. Uma possível explicação para esse fenômeno seria o cenário competitivo o qual os hospitais privados estão inseridos.

\section{CONSIDERAÇÕES FINAIS}

Durante a realização do artigo assumiu-se o desafio em se buscar conhecimento da atual situação do Brasil no quesito relação existente entre assistência farmacêutica hospitalar e o processo de acreditação.

Ao concluir esse documento pudemos considerar que a Acreditação Hospitalar é um tema de grande relevância quando se trata de farmácia hospitalar principalmente no âmbito da assistência farmacêutica já que através dela constata-se que as boas práticas da qualidade em serviços de saúde nos hospitais, quando associada à acreditação, proporcionam inúmeros benefícios. Outro fato a ser considerado, é que deve haver incentivos para que os hospitais se interessem em se acreditarem já que o processo é voluntário e caro, baseado no fato de que isso será de grande relevância tanto para a unidade de tratamento quanto para o paciente.

\section{REFERÊNCIAS}

ALONSO, LUIZA BETH NUNES; DROVAL, CLARISSE; FERNEDA, EDILSON; EMÍDIO, LUCI. Acreditação hospitalar e a gestão da qualidade dos processos assistenciais. Perspectivas em Gestão \& Conhecimento, v. 4, n. 2, p. 34-49, 2014.

ANVISA. Acreditação: a busca pela qualidade nos serviços de saúde. Rev. Saúde Pública, São Paulo, v. 38, n. 2, Abr. 2004.

ANVISA. Sistema Brasileiro de Acreditação. Disponível em: <http://www.anvisa.gov.br/servicosaude/acreditacao/entrevista.htm>. Acesso em: 13 jun. 2019.

BRASIL. MINISTÉRIO DA SAÚDE. MANUAL DAS ORGANIZAÇÕES PRESTADORAS DE SERVIÇOS HOSPITALARES. Disponível em: < 
http://bvsms.saude.gov.br/bvs/publicacoes/MH_completo.pdf>. Acesso em: 12 jun. 2019.

BRASIL. MINISTÉRIO DA SAÚDE. PORTARIA № 4.283, DE 30 DE DEZEMBRO DE 2010. Aprova as diretrizes e estratégias para organização, fortalecimento e aprimoramento das ações e serviços de farmácia no âmbito dos hospitais. Disponível em: <http://bvsms.saude.gov.br/bvs/saudelegis/gm/2010/prt4283_30_12_2010.html>. Acesso em 16 jun. 2019.

BRASIL. MINISTÉRIO DA SAÚDE. RESOLUÇÃO № 338, DE 06 DE MAIO DE 2004. Aprova a Política Nacional de Assistência Farmacêutica. Disponível em: <http://bvsms.saude.gov.br/bvs/saudelegis/cns/2004/res0338_06_05_2004.html>. Acesso em: 12 jun. 2019.

CFF - CONSELHO FEDERAL DE FARMÁCIA. RESOLUÇÃO N 572, de 25 de abril de 2013. Disponível em: <http://www.cff.org.br/pagina.php?id=14\&titulo=>. Acesso em: 17 jun. 2019.

CBA - CONSÓRCIO BRASILEIRO DE ACREDITAÇÃO. Disponível em: < https://cbacred.org.br/site/o-cba/>. Acesso em: 19 mar. 2020.

EBSERH - EMPRESA BRASILEIRA DE SERVIÇOS HOSPITALARES. Disponível em: $<$ http://www2.ebserh.gov.br/web/hc-ufmg/acreditacao-hospitalar>. Acesso em: 16 jun. 2019.

FOLHA DE SÃO PAULO. Só 5\% dos hospitais no Brasil têm selo de qualidade; $40 \%$ deles estão em São Paulo. 2015. Disponível em: $<$ https://www1.folha.uol.com.br/seminariosfolha/2015/05/1628532-so-5-doshospitais-do-brasil-tem-selo-de-qualidade-40-deles-estao-em-sp.shtml>. Acesso em: 13 jun. 2019.

FORTES, MARIA THEREZA RIBEIRO; FARIA, TATIANA WARGAS. Acreditação: ferramenta ou política para organização dos sistemas de saúde? Acta Paul Enferm; 25(4):626-31 2012. 
GUIA DA FARMÁCIA. Farmacêutico: um profissional multitarefa. 2018. Disponível em: $\quad<$ https://guiadafarmacia.com.br/materia/farmaceutico-um-profissionalmultitarefa/>. Acesso em: 12 jun. 2019.

ICTQ - INSTITUTO DE CIÊNCIA, TECNOLOGIA E QUALIDADE. Disponível em: $<$ https://www.ictq.com.br/guia-de-carreiras/295-a-carreira-do-farmaceuticohospitalar>. Acesso em: 12 jun. 2019.

MV. Conheça as principais acreditações e certificações hospitalares. 2018. Disponível e: <http://www.mv.com.br/pt/blog/conheca-as-principais-acreditacoes-e-certificacoeshospitalares>. Acesso em: 19 mar. 2020.

ONA - ORGANIZAÇÃO NACIONAL DE ACREDITAÇÃO. Disponível em: $<$ https://www.ona.org.br/acreditacao/o-que-e-acreditacaos. Acesso em: 19 mar. 2020.

ONA - ORGANIZAÇÃO NACIONAL DE ACREDITAÇÃO. Disponível em: $<$ https://www.ona.org.br/uploads/TABELAS_DE_TAXAS_PARA_CERTIFICACAO_2. pdf >. Acesso em: 19 mar. 2020.

ONA - ORGANIZAÇÃO NACIONAL DE ACREDITAÇÃO. Disponível em: $<$ https://www.ona.org.br/mapa-de-acreditacoes>. Acesso em: 02 mar. 2020.

RODRIGUES, MARIA LUCIA.; TUMA, ILENIR LEÃO. Certificação em Farmácia Hospitalar. Pharmacia Brasileira (encarte). Brasília: CFF, 2011. Disponível em: < http://www.cff.org.br/sistemas/geral/revista/pdf/132/encarte_farmAcia_hospitalar.pdf. > Acesso em: 19 mar. 2020.

ROSA, GERMANO MENDES.; TOLEDO, JOSÉ CARLOS DE. Certificações e acreditações buscadas por hospitais brasileiros. Encontro Interestadual de Engenharia de Produção, 2015. 
SACK, C. et al. Is there an association between hospital accreditation and patient satisfaction with hospital care? A survey of 370000 patients treated by 73 hospitals. International Journal for Quality in Health Care, v. 23, n. 3, p. 278-283, 2011.

SANTOS, JAQUELINE ALVES DE; LIMBERGER, JANE BEATRIZ. Indicadores de avaliação da assistência farmacêutica na acreditação hospitalar. Revista de Administração em Saúde- Vol. 18, № 70, jan. - mar. 2018.

SITEWARE. Disponível em: <https://www.siteware.com.br/qualidade/indicadoresdesempenho-farmacia-hospitalar/>. Acesso em: 26 mar. 2020.

SOCIEDADE BRASILEIRA DE FARMÁCIA HOSPITALAR - SBRAFH. Padrões mínimos para farmácia hospitalar. $3^{\text {a }}$ edição. Goiânia - Belo Horizonte: SBRAFH, 2017. Disponível em: <http://www.sbrafh.org.br/site/public/docs/padroes.pdf >. Acesso em: 19 mar. 2020.

SHAW, CHARLES D. External quality mechanisms for health care: summary of the ExPeRT Project on visitatie, accreditation, EFQM and ISO Assessment in European Union Countries. Int J Qual Health Care;12(3):169-75, 2000.

Enviado: Abril, 2020.

Aprovado: Maio, 2020. 